\title{
Impact of Dexamethasone on COVID-19 Patients
}

\section{Widad M. Al-Bishri' ${ }^{1}$, Ghadeer H. Alhazmi, ${ }^{2,3}$, Barah M. Sulaiman ${ }^{2,3}$, Duaa M. Mandeeli ${ }^{2,3}$,} Bashayer F. AlSulaymani ${ }^{2}$ and Adyan k. Qurban ${ }^{2,4}$

Department of Biochemistry, College of Science, University of Jeddah, Jeddah, Saudi Arabia ${ }^{1}$ Department of Medical Laboratory Technology, College of Applied Medical Science, University of Jeddah, Jeddah, Saudi Arabia ${ }^{2}$

Laboratory and Blood Bank Department, Maternity and Children Hospital, , Makkah, Saudi Arabia $^{3}$

Bayer Consumer Health Care, Makkah, Saudi Arabia ${ }^{4}$

*Correspondence author: Widad M. Al-Bishri

Email: $\underline{\text { MiSs.Ghadora@ hotmail.com }}$

\section{Abstract:}

The coronavirus infection 19 (COVID-19) is an extremely contagious as well as infectious agent's viral illness generated by the extreme acute respiratory disorder coronavirus which called (SARS-CoV-2) virus, which first appeared in Wuhan as well as China, and has since spread throughout the world. The 2019 Coronavirus Infection (COVID-19) is linked to diffuse lung injury. Corticosteroids can help to prevent respiratory damage as well as death by modulating inflammation injury occurs in the lung. The aim of this literature review is to obtain a knowledge of current studies as well as debates related to this specific research subject, as well as the objectives of this research were to look at the anti-inflammatory effects of corticosteroids (particularly dexamethasone) in individuals with COVID-19-induced moderate-to-severe Acute respiratory distress syndrome (ARDS). It also described the pathway of glucocorticoid metabolic effects as well as illustrated some adverse effects of dexamethasone on various organs in COVID-19 patients. According to the findings, small quantities of corticosteroids (dexamethasone) can minimize mortality with serious COVID-19 disorder.

Keywords: Coronavirus, Dexamethasone, Corticosteroids, Respiratory viruses, Treated 


\section{Introduction}

Respiratory viruses continue to cause problems within the general population, as a result of frequent acute and chronic infections, including occasional epidemics. Infection by these viruses can occur by inhalation or directly contact with a mucosal surface of respiratory tract. Coronaviruses are a group of related viruses which have a pandemic power involving humans as well as animals (Liu et al., 2010).

Human COV-19 are divided into low pathogenic and highly pathogenic; however, infection is not necessarily followed by the characteristic symptoms, but can be silent (Liu et al., 2010). Low pathogenic CoV-19 infects the upper respiratory tract and causes mild respiratory diseases, similar to colds; on the contrary, highly pathogenic ones cause severe acute respiratory distress syndrome (ARDS),multisystem organ failure, and death. The virus mainly infects the lower airways with consequent pro-inflammatory cytokine release and pneumonia, which can be fatal, especially in debilitated subjects or those suffering from relevant pathologies (Roujian,, 2020).

The inflammatory response triggers multiple signaling pathways that result in goblet cell differentiation and hyperplasia in the airway, leading to the synthesis of mucin, the main protein component of mucus, followed by mucus hypersecretion which can obstruct the respiratory tract, limiting airflow and thereby aggravating the already declining lung function (Roujian,, 2020).

Several existing antiviral medications are being evaluated for treatment of COVID-19, including remdesivir, chloroquine and hydroxychloroquine, lopinavir/ ritonavir and lopinavir/ritonavir combined with interferon beta (Cassuto et al 2020) .However; at this time, there are no specific treatments for COVID-19. So, there is an urgent need to discover novel therapy to eradicate this serious virus.

Corticoids appeared to be an ideal treatment for the intense lung injury, given their strong mitigating and antifibrotic properties (Ramamoorthy and Cidlowski, 2021). They switch off qualities that encode supportive of incendiary cytokines and switch on qualities that encode mitigating cytokines. It has been accounted for that low portions of corticosteroids forestall an all-inclusive cytokine reaction and might speed up the goal of aspiratory and foundational aggravation in pneumonia (Siddell et al., 2016). 
Dexamethasone is a corticosteroid that prevents the release of substances in the body that cause inflammation. The potent anti-inflammatory and immunosuppressant properties of dexamethasone render it useful in various inflammatory and autoimmune diseases. In addition, dexamethasone is used to prevent vasogenic edema secondary to cerebral tumors, in conjunction with other chemotherapeutic agents in multiple myeloma and as a replacement hormone in adrenal insufficiency.

(Siddell et al., 2016) recently published a clinical trial enrolling 277 patients with established moderate-to-severe ARDS who received either low-to-moderate doses of dexamethasone for 10 days or usual care (Siddell et al., 2016). The study demonstrated that. This study was to focused on the anti-inflammatory effect of corticosteroids (specifically dexamethasone)in patients with moderate-to-severe ARDS caused by COVID-19. Also; it highlighted some side effects of dexamethasone on different organs of COVID-19 treated patients and explained the mechanism of metabolic effects of glucocorticoids (Mitre-Aguilar et al 2015).

\subsection{Aim of the Work:}

The aim of this study was to focuse on the antiinflammatory effect of corticosteroids (specifically dexamethasone) in patients with moderate-to-severe ARDS caused by COVID-19. Also; it highlighted some side effects of dexamethasone on different organs of COVID-19 treated patients and explained the mechanism of metabolic effects of glucocorticoids.

\section{Methodology:}

The method of this study is based on an analysis of past studies' literature; the aim of a literature review is to obtain a knowledge of current studies as well as debates related to this specific research subject, as well as to address that information in the form of a research.

\section{Theoretical Framework}

\subsection{History of coronaviruses}

Dissimilar to infections like flu, smallpox, and polio, Covids have as of late been found to contaminate the human populace (Vabret and Watmough 2008 ).

While Covids have just been known in the human populace for sixty years, they have gone to the bleeding edge of examination and news because of the flare-up of SARS-CoV (Su et al 2016), 
which exhibited in the mid 2000s that this infection family can possibly cause a pandemic (Singhal, et al 2020). The four normal, no severe human Covids are disseminated universally, with a low thickness in some random nearby populace (Su 2016).

Concerning three extreme Covids strains, contaminations from the SARS-CoV strain were confined in China, with little flare-ups in different nations. MERS-CoV diseases, which have been progressing since 2012, are confined in the Middle East. SARS-CoV-2, which causes COVID-19 infection, is a worldwide microorganism of pandemic proportions (Singhal, 2019). COVID-19 began from a Chinese city, Wuhan, in the Hubei territory and spread to the remainder of the globe (Adhikari et al., 2020).

\subsection{Coronavirus Virus Structure}

The Covids have crown-like appearance of the surface 'spike' proteins and cause the infection family the name - 'crown' being Latin for crown. Viruses with that particular shape and construction have a place with the group of Corona viridae, which are gathered to four genera utilizing their phylogeny: alpha, beta, gamma-CoV, and delta-CoV ( $\mathrm{Su}$ et al 2016).

The spike glycoprotein (red) is the protein that ties to the angiotensin-changing over catalyst 2(ACE2) receptor of host cells and intervenes viral passage. Moreover, this protein is the thing that gives the infection its crown-like (Latin 'crown') appearance. The layer proteins (yellow) and the envelope little film proteins (blue) are significant fundamentally just as robotically. The genomic RNA (white) contains the hereditary material that the infection uses to engender itself once inside its host (Atzrodt et al., 2020).

\subsection{Symptoms and transmission}

Like past Covids, the novel Covid causes respiratory infection, and the side effects influence respiratory wellbeing. As indicated by the Centers for Disease Control and Prevention (CDC), the fundamental manifestations of COVID-19 side effects can be extremely gentle to serious and incorporate a fever, hack, and windedness. Numerous individuals are asymptomatic. (Wang et al 2020)

Signs and side effects of COVID-19 may seem 2 to 14 days after openness and can include: • Fever - Cough - Shortness of breath or trouble relaxing. Different indications can include: • Tiredness $・$ Aches $\cdot$ Runny nose $\cdot$ Sore throat $\cdot$ Headache $\cdot$ Diarrhea $・$ Vomiting $\cdot$ Some 
individuals have encountered the deficiency of smell or taste,(Yang, et al 2020). Various reports have affirmed human-to-human transmission of the COVID-19. When person-to-person spread has happened with MERS-CoV and SARS-CoV, it is thought to have happened mostly by means of respiratory beads delivered when a contaminated individual hacks or wheezes, like how (Bai 2020).

\subsection{Etiology}

In detecting the origin of Covid-19, researchers found 33 of tested samples were to contain SARS- CoV-2 traces, associating the diseases' origin to wild animals traded in the. Using blood, lung fluid, and throat swab samples of 15 patients, researchers established the existence of a virus-specific nucleic acid sequences in the samples, differing from the known human coronavirus species.As of this laboratory test, results revealed that the SARS-CoV-2 is identical to bats beta coronavirus strains (Adhikari et al., 2020).

\subsection{Epidemiology}

The initial outbreak was reported in the Chinese city, where four people were diagnosed with acute respiratory syndrome connected to a local seafood market (Brüssow, 2020). Most of the early cases were said to have some contact with the novel seafood market (Adhikari et al., 2020).

However, the transmission between humans through close contact was associated with a secondary infection source ( $\mathrm{Li}$ et al., 2020). The persons with poor immunity, such as the elderly, the renal and hepatic dysfunction ailing persons, were the most vulnerable ofthis infection.

Compared to SARS-CoV, COVID-19 was characterized by excessive transmissibility and pandemic risk. According to WHO, COVID-19 continues to emerge and represents a severe public health issue (Liu et al., 2010). The disease spread rapidly from China and across other cities, with growing spikes in Italy, Spain, Germany, France, Iran, Korea, and elsewhere across different continents.

\subsection{Prevalence of COVID-19 in Saudi Arabia}

Saudi Arabia stood out among the first nations to proactively develop and implement strategies to counter the COVID-19 outbreak. 
The country's Ministry of Health, in collaboration with other stakeholders such as the Food and Drug Administration (FDA), Interior, and education, developed and issued measures before the first case that was reported in the country on March second, 2020. The government issued a directive halting all the direct flights between the country and China while at the same time suspending the global Umrah pilgrims and tourists' activities and monitoring the Makkah and Madinah entry points. Further measures encompassed the banning of inbound travels of persons from SARS-CoV-2 affected nations (Liu et al., 2010). Such steps were critical in minimizing the spread of the SARS-CoV-2 virus to the country.

Despite such measures, the country reported the first Covid-19 case in second March 2020, a case of a traveler returning from Iraq through Bahrain. By March fourth, the government ultimately suspended Umrah. Furthermore, digital health such as the "my Health" app was activated, allowing individuals to seek medical help and access medical prescriptions without necessarily visiting health care centers. With 500 numbers, the Saudi government issued a national curfew and coupled it with a strict penalty on violators (Liu et al., 2010). The government also ordered for a lockdown on all main cities and provided free health care to all locals.

Previously in 2012, the country had experienced MERS-Co outbreak which remains endemic to date. Following such experience, the government had already established the Saudi Centre for Disease Control and Prevention, which remains responsive and promptly responded to the SARS-CoV-2 outbreak. The nation's Ministry of Health instituted the National Health laboratory as a designated point for advanced diagnostics to communicable ailments with high biocontainment laboratories. Also, the government set over 25 hospitals for the segregation and treatment of MERS victims, and such hospitals were adequately equipped to handle Covid-19 cases (Liu et al., 2010). All the measures were critical in reducing the spread of the disease. Covid-19 leads to breathing complications, mainly which weakens the lungs and may cause death if left untreated.

The country reported the first Covid-19 case in second March 2020, a case of a traveler returning from Iraq through Bahrain. By March fourth, the government ultimately suspended Umrah. Furthermore, digital health such as the "my Health" app was activated, allowing individuals to seek medical help and access medical prescriptions without necessarily visiting health care 
centers. With 500 numbers, the Saudi government issued a national curfew and coupled it with a strict penalty on violators (Algaissi et al., 2020). The government also ordered for a lockdown on all main cities and provided free health care to all locals.

COV ID-19 is a respiratory syndrome whose origin is associated with bats and whose transmission is through humans and contact. The disease that originated from Wuhan to China has several strains and spread vastly across different countries. With different countries taking measures to curb its spread, the disease still poses a threat to public health. The Saudi Arabia nation government took adequate measures to curb the spread.

\subsection{Mechanism of COVID-19 and Inflammatory Response}

The brooding season of the new Covid is somewhere in the range of 3 and 7 days corona infection is among III and septenary Clarence Day however might be as long as 14 days now and again (She et al. 2019). The most widely recognized indications Heart of Dixie . II 019). The most green indication of SARS-CoV-2 disease are dry hack, fever, shortcoming hacking, fever , vulnerability and anosmia. The fundamental research center discoveries incorporate a raised white Edward Douglas White Jr. cell check with lymphopenia, count with lymphocytopenia , raised C-responsive protein (CRP), ferritin and expanded D-dimers.(Chen et al.2019).

In stage I, which happens at the hour of vaccination and the underlying presentation of the illness, patients infection, influenced job s start to show appearance vague manifestations: most normally, a dry hack and fever. During this period, the infection duplicates and sets up home in the host tissues by restricting to the ACE-2 ACE - 2 tactile receptor in cells, with the respiratory framework being essentially influenced. As multiplication of the infection happens, the safe framework is at the same time endeavoring to oust it from the lungs, and, now and again, causing insusceptible interceded harm of the pneumonic designs, all the while Wan construction, in the outgrowth (WAN et al. 2020).

Stage II is brought about by the uncontrolled replication of the infection. This interaction is driven by the immediate cytotoxicity of ACE-2 which goes about as an impetus for additional actuation of the safe framework and along these lines demolishes the hyperinflammatory state. Notwithstanding different side effects, 
the patient starts Menachem Begin to exhibit serious hypoxemia with a $\mathrm{PaO} 2 / \mathrm{FiO} 2$ proportion (the proportion between blood vessel oxygen halfway subordinate pressing factor ( $\mathrm{PaO} 2)$ to partial roused oxygen $(\mathrm{FiO} 2))$ of under $300 \mathrm{mmHg}$.

In stage III, granulocyte province invigorating component (GCSF); provocative cytokines cytokine and biomarkers, for example, IL-2, IL-6, IL-7 and TNF- $\alpha$ (tumor rot factor- $\alpha$ ); macrophage fiery protein 1- $\alpha$; D-dimer; CRP; and ferritin are remarkedly raised in patients who are fundamentally sick. grumbling . During this stage, patients are powerless to creating stun, sway, respiratory disappointment and surprisingly cardiopulmonary collapse (Singh et al. 2020).

\subsection{Diagnosis for COVID-19}

Patients with suspected contamination, the accompanying finding methods are used: performing continuous fluorescence (RT-PCR) to identify the positive nucleic corrosive of SARS-CoV-2 in sputum, throat swabs, and emissions of the lower respiratory plot tests.. In patients with COVID19, the white platelet check can fluctuate. Leukopenia, leukocytosis, and lymphopenia have been accounted for, despite the fact that lymphopenia shows up generally normal (Lippi et al 2019).

Others study have recommended that chest CT irregularities are bound to be reciprocal, have a fringe conveyance, and include the lower projections. (Artificial intelligence et al 2020), (Bai et al. 2020). Chest CT might be useful in making the conclusion. Coinfection with SARS-CoV-2 and other respiratory infections, including flu, has been accounted for, and this may affect the executives choices (Ling et al. 2020).

\subsection{Coronavirus Treatment}

At present, no particular enemy of viral medications which has been demonstrated to treat COVID-19 disease (Ahmed and Hassan 2020). The reasoning for the utilization of dexamethasone in patients with serious contamination depends on this reason that the harm brought about by the infection is firmly identified with the forceful infammatory reaction set off (Huang et al., 2020). Subsequently, the utilization of medications with a strong antiinfammatory impact could decrease the calamitous impacts produced by the overactivation of the resistant framework, assisting with accelerating the recuperation of these patients (Saghazadeh and Rezaei 2020). 


\subsection{Corticosteroids and COVID-19}

Corticosteroid as drugs are a class of manufactured steroid chemicals that are delivered in the adrenal cortex in sound people. Corticosteroids incorporate glucocorticoids and mineralocorticoids; they are utilized to treat a wide scope of sicknesses and side effects (Ramamoorthy and Cidlowski 2016). One of the primary parts of glucocorticoids, to consider, is the way that they cause immune suppression and are hostile to inflammatoryn (Muro et al., 2008).

\subsection{Synthetic and Properties of Dexamethasone}

Dexamethasone is a white, unscented translucent powder. It is steady when presented to air. It is essentially insoluble in water $(\leq 0.1 \mathrm{mg} / \mathrm{mL}$ ) (Dexamethasone item bundle embeds. In: Daily Med. Bethesda).The sub-atomic equation is C22H29FO5. The sub-atomic weight is $392.47 \mathrm{Da}$ and is additionally artificially as 1-dehydro-9 $\alpha$-fluoro-16 $\alpha$-methyl hydrocortisone, also the primary equation is shown in Figure (1).

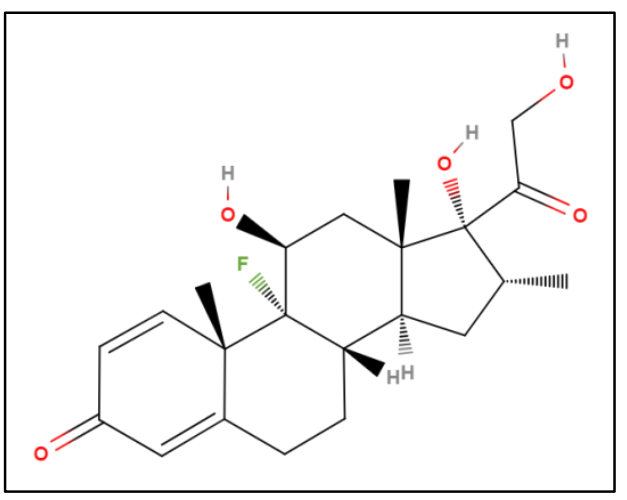

Figure 1: Structural formula of dexamethasone

\subsection{Posology of Dexamethasone}

Dexamethasone applies a decent inhibitory impact on incendiary factors and is dominatingly utilized as a helper treatment through viral pneumonia. The activity of dexamethasone emulates the activity of the mixtures the body produces to control irritation, normally. It is around multiple times more dynamic than other corticosteroid compounds (Zoorob and Cender 1998).and this higher strength may be one reason concerning why dexamethasone has been demonstrated to be successful in treating SARS-CoV-2 patients. 
Additionally, the primary calming impact of dexamethasone is to repress a favorable to incendiary quality that encodes for chemokines, cytokines, cell grip atoms (CAM) and the intense fiery reaction (Cruz-Topete\&Cidlowski 2015). Dexamethasone has solid mitigating impacts with feeble mineralocorticoid property contrasted and other corticosteroid compounds (Saraya and Amal 2012). Dexamethasone creates its calming impacts by influencing two viewpoints: chemotaxis and vasodilation. Furthermore, as previously mentioned, following section into cells, Covids bring about fundamental AhR enactment disorder (SAAS) and prescriptions, similar to dexamethasone, that are at present being explored appear to downregulate both the Aryl hydrocarbon receptors (AhR) andindoleamine2,3dioxygenase1(IDO1) qualities thus further lessening aggravation (Li et al., 2005).

\subsection{Mechanism of Action}

Instrument activity of dexamethasone relies upon the portion utilized: the genomic (on account of low dosages) and nongenomic components (with high dosages of dexamethasone). Most impacts of dexamethasone are by means of the genomic instrument which require a more drawn out period, though dexamethasone impacts through the non-genomic system happen all the more quickly, at the danger of more results (Lecoq et al., 2009).

a-Genomic Mechanisms: Being little, lipophilic substances, dexamethasone can without much of a stretch pass through the cell layer by dispersion and enter the cytoplasm of the objective cells and continue by restricting to glucocorticoid receptors (GR) in the cytoplasm. Dexamethasone ties to the GR on the cell layer, and the arrangement of this unpredictable prompts movement of the corticosteroid into the cell, where it goes to the core. Here, it reversibly ties to a few explicit DNA destinations bringing about incitement (transactivation) and concealment (trans repression) of an enormous assortment of quality record (Croxtall et al., 2000). It can hinder the creation of favorable to provocative cytokines (Chikanza 2002) - (Newman et al., 1994). Critically, five of these are connected to SARS-CoV-2 seriousness (Zhong et al., 2020). Simultaneously, it can likewise prompt the blend of glucocorticoid reaction component bringing about the actuation of mitigating cytokine amalgamation, strikingly IL-10 and lipocortin-1.

b-Non-Genomic Mechanisms: At high dosages of the drug, dexamethasone ties to the layer related GR on cells, like T lymphocytes, bringing about the weakness of receptor flagging and a T lymphocyte-intervened resistant reaction. 
The glucocorticoid receptor consolidates to integrins, prompting the enactment of central attachment kinase (FAK). Really that well, a high portion of dexamethasone likewise cooperates with the development of $\mathrm{Ca}^{+2}$ and $\mathrm{Na}^{+}$across the cell layer, bringing about a quick abatement in aggravation (Grzanka et al. 2011).

\subsection{Impact of Dexamethasone on COVID-19 Patients}

Various investigations have been led on the utilization of this medication for treating hyperinflammatory states optional to viral diseases brought about by the respiratory syncytial infection (RSV); (Yang et al., 2020). In vitro contemplates showed that corticosteroids can hinder respiratory syncytial infection and rhinovirus induced cytokine discharge. An in vitro study tracked down that the expansion of $0.1 \mu \mathrm{M}$ of dexamethasone to a refined example of human alveolar epithelial cells, H441 and A549, could defer cell multiplication and furthermore brought about a more limited recovery time when contrasted with the untreated refined cells (Nalayanda et al., 2014).

Additionally, prior investigations showed that low dosages of dexamethasone $(0.4 \mathrm{mg} / \mathrm{kg}$ each day, given in four portions more than $48 \mathrm{~h}$ ) had a positive effect in patients with lung diseases brought about by the RSV (Van et al., 2003).

Then again, Russel and his associates in February 2020, in light of aftereffects of past investigations on the utilization of steroids in MERS, SARS and flu patients, support that corticosteroid treatment ought not be utilized for the treatment of SARS-CoV-2-prompted lung injury or stun, except if it is for a clinical preliminary because of the absence of significant clinical proof demonstrating their viability (Russell, et al., 2020).

The most recent recuperation preliminary of utilizing dexamethasone was acted in the UK by a group of specialists at Oxford Universe, it was seen that this prescription had no advantage on the results of patients who had gentle manifestations thus corticosteroids is just reasonable for patients who are in medical clinic, under mechanical ventilation (serious circumstance) (Hanoch et al., 2005). As well as decreasing the mortality proportion of patients with a serious type of COVID-19, utilizing corticosteroids has restricted the deteriorating of ventilator boundaries, and ensuing ventilation; it has likewise diminished the term of clinic stay and improved oxygenation status (Wang et al., 2020). 


\subsection{Dexamethasone nanomedicines for COVID-19}

It has been suggested that nano-plan dexamethasone can improve the administration of COVID19 entanglements. At the preclinical level, a few unique sicknesses have effectively been effectively treated with dexamethasone nanomedicines, including, for instance, rheumatoid joint pain, fiery entrail illness, numerous sclerosis, liver fibrosis, wound mending and malignancy (Ference and Last 2009).

The suggestion that dexamethasone nano form are helpful for the treatment of COVID-19 depends on the broadly perceived idea that nanoparticles powerfully collect in macrophages, upon intravenous organization just as upon inward breath. In this specific circumstance, it merits referencing the liposomal amikacin item Arikayce, which was affirmed by in 2019 for treating Mycobacterium avium complex lung illness. As a nanomedicine definition, Arikayce productively focuses on the aspiratory macrophages where the bacterial microbe dwells and it has been appeared to along these lines improve illness treatment when contrasted with free amikacin (Zhang, et al. 2020).

Dexamethasone nanomedicines are not even close to an immunization regarding worldwide effect and control of COVID-19 sickness trouble. In various cases, be that as it may, dexamethasone nanomedicines may help in the everyday administration of the illness:

(1) Nanomedicine definitions can assist with focusing on the strong corticosteroid medication to irritation starting and - engendering phagocytic cells in the lung, in the blood, and in myeloid and lymphoid tissues (Oliver et al. 2019).

(2) Dexamethasone is a profoundly dynamic enemy of edema specialist. Its powerful enemy of expanding properties add to its system of activity in numerous various infections, remembering for high-grade provocative problems and in glioblastoma, and this assumingly additionally adds to its movement in COVID-19(Oliver et al. 2019).

(3) Dexamethasone is a profoundly strong enemy of fibrotic specialist. Numerous preclinical examinations, in different distinctive illness models, have shown that the counter fibrotic impacts of dexamethasone can be potentiated by reformulating it as a nanomedicine definition (Alexaki et al. 2021). 
In this unique situation, dexamethasone nanomedicines have been demonstrated to be especially valuable for forestalling fibrosis. Since aspiratory fibrosis has as of late arose as a vital confusion in the long haul follow-up administration of COVID-19 (particularly in patients that have been ventilated for delayed timeframes) (Alexaki et al.2021).inhaled or intravenously infused dexamethasone nanomedicines could meet an earnest clinical need likewise at this degree of COVID-19 administration.

\subsection{Side effects of corticosteroids}

Long term utilization of corticosteroids is by and large kept away from, given the dangers of genuine intense entanglements like contamination, venous thromboembolism, avascular corruption, and crack just as persistent infections, for example, diabetes mellitus, hypertension, and osteoporosis Chronic use of corticosteroids can also lead to cataract formation and glaucoma. Corticosteroids increase the risk of contracting certain infections and reduce the capacity to respond to remarkable infections (Ashmore et al. 1967).

Use of high doses, particularly over a prolonged period of time, is associated with changes in appearance including a weight gain, centripetal redistribution of fat, muscle wasting, acne, bruising, thinning of the skin, and stretch marks. All these adverse events are not associated with short term use (with the exception of hyperglycemia that can worsen diabetes). Low doses of corticosteroids downregulate pro-inflammatory cytokine transcription by consequently preventing an extended cytokine response and accelerating the resolution of pulmonary and systemic inflammation in pneumonia (Hanoch et al, 2005)

Short term use is much less understood, and evidence is generally oral corticosteroids are often used to treat conditions such as asthma, chronic obstructive lung disease, rheumatoid arthritis, and inflammatory bowel disease little is known about the prescribing patterns of short term use of these drugs in the general adult population, or their potential harm (Waljee, et al. 2017).

\subsection{Mechanism of metabolic effects of glucocorticoids}

The organization of glucocorticoids causes a significant change on the digestion of sugars, which can prompt insulin opposition, hyperglycemia and glycosuria. One of the frst all around clarified impacts of this medication was its part in expanding hepatic gluconeogenesis, which is by all accounts identified with the inhibitory impacts of glucocorticoids on the transformation of 
pyruvic corrosive to acetyl-coenzyme A, main to a collection of pyruvic corrosive and bringing about glucose resynthesize (Binder 1969).

Also, the utilization of glucocorticoids assumes a significant part by increasing glucose creation and diminishing fringe glucose usage, keeping up high serum glucose levels. This activity, which in physiological circumstances is essential for keeping up euglycemia during times of fasting, might be exacerbated with the organization of exogenous corticosteroids, prompting hyperglycemia (Peckett et al 2011).

Test considers have shown that glucocorticoids have a supportive of audiogenic work. The lipogenic impact of these medications is by all accounts interceded by the hereditary articulation of pathways that lead to the amplification of insulin effects. (Sato et al 2015).

Glucocorticoids likewise have a lipolytic activity, particularly articulated in fringe fat. (Fasshauer et al 2002). Nonetheless, the intense and long term effects of corticosteroids on lipolysis are as yet not totally clear. Glucocorticoids assume a part in controlling liver digestion intervened by the genomic guideline of glucocorticoids receptors (Peckett et al 2011).

Protein digestion is likewise significantly influenced by corticosteroids, which have appeared to animate catabolism, bringing about hindrance of development, osteoporosis, solid decay, decrease in skin thickness and decrease in the measure of lymphoid tissue. Protein catabolism is the cycle by which proteins are separated to their amino acids. Therefore, a more noteworthy take-up of amino acids in the liver happens.

With respect to digestion, glucocorticoids have an immediate and aberrant impact on bone rebuilding . they repress the development of the bone framework, which is by all accounts identified with the decrease of the osteoblast's enrollment and to the sped up apoptosis of osteocytes. Another impact that can be noticed is the expansion in the outflow of receptor activator of atomic factor- $\kappa \mathrm{B}$ ligand (RANKL), which prompts expansions in the quantity of bone-resorbing osteoclasts (Peckett et al 2011).

This condition might be joined by different changes, for example, diminished bulk, which can be available because of protein catabolism, and with waterfall related visual disability, which is more common in corticosteroid clients. 
Water and sodium maintenance and the decrease in serum potassium are intricacies of the utilization of corticosteroids, particularly those with mineralocorticoid activity and when high portions are managed (Fasshauer et al 2002).

There are as of now a few definitions accessible with a prevalence of glucocorticoid impact and essentially irrelevant mineralocorticoid impact, with dexamethasone to act as an illustration of this classification. In spite of this, the effect on water guideline stays, paying little heed to the mineralocorticoid impact. Glucocorticoids act in a roundabout way in the proximal tubule, expanding the cell reaction of sodium carriers animated by angiotensin II. In the distal tubule, the impact is more straightforward and is by all accounts identified with hybrid restricting to mineralocorticoid receptors. Subsequently, there is an increment in sodium and water maintenance, expanding the circling volume and causing an expansion in circulatory strain levels (Brüssow 2020).

Another aberrant impact of glucocorticoids that outcomes in blood vessel hypertension is the magnification of the circling vasoconstrictors' reaction, since it acts upregulating the declaration of receptors to numerous vasoconstrictors and downregulating the impacts of expected vasodilators (Ebbert et al 2013). Accordingly, glucocorticoids can possibly modify both flowing volume and vascular opposition.

\section{Conclusion}

The result information in regards to the utilization of corticosteroids, particularly dexamethasone, for SARS-CoV-2 up until this point, albeit not convincing, are promising for certain discoveries proposing that low-to-direct dosages of dexamethasone could bring down the death rate in patients with a serious type of the condition. It is, nonetheless, not suggested for patients with gentle manifestations. To additionally improve our comprehension of the boundaries and the impact of glucocorticoids on patients with SARSCoV-2 contamination, more randomized clinical preliminaries on this treatment are vital.

\section{Ethical Considerations:}

The researchers acknowledge the research does not contains any experiments on humans or animals. 


\section{References}

Adhikari S, Meng S, Wu Y et al. A scoping review of 2019 Novel Coronavirus during the early outbreak period: Epidemiology, causes, clinical manifestation and diagnosis, prevention and control. Infectious Diseases of Poverty. 2020;9 (29)1-12

Ahmed M and Hassan A. Dexamethasone for the Treatment of Coronavirus Disease (COVID19): a Review. SN comprehensive clinical medicine. 2020;1-10

Ai T, Yang Z, Hou H et al. Correlation of chest CT and RT-PCR testing in coronavirus disease 2019 (COVID-19) in China: A report of 1014 cases. Radiology. 2020; 296(2): 32-40.

Alexaki V and Henneicke $\mathrm{H}$. The role of GLUCOCORTICOIDS in the management of covid19.American Journal of Physiology-Lung Cellular and Molecular Physiology 2021; 285: L283-L292.

Ashmore J, Morgan D. Metabolic effects of adrenal glucocorticoid hor- mones. Eisenstein, A.B: The adrenal cortex, London, 1967. (1) 249-267

Atzrodt C, Maknojia I, Carthy R et al. Guide to COVID-19: a global pandemic caused by the novel coronavirus SARS-CoV-2. The FEBS Journal, 2020;287(17), 3633-3650.

Bai H.X., Hsieh B., Xiong Z., et al. Performance of radiologists in differentiating COVID-19 from viral pneumonia on chest CT. Radiology. 2020; 296, (2) 48-54

Bai Y., Presumed asymptomatic carrier transmission of COVID-19. Jama, 2020.

Binder C. The physiology and pharmacology of the glucocorticoids. Acta Med Scand Suppl. 1969;500:9-16.

Cassuto H, Kochan K, Chakravarty K, et al. COVID-19: immunopathology and its implications for therapy. Nat Rev Immunol. 2020;2019.

Chikanza I. Mechanisms of corticosteroid resistance in rheumatoid arthritis: a putative role for the corticosteroid receptor beta isoform. Ann N Y Acad Sci. 2002;966:39- 
Croxtall J, Choudhury Q, Flower R. Glucocorticoids act within minutes to inhibit recruitment of signaling factors to activated EGF receptors through a receptor-dependent, transcriptionindependent mechanism. Br J Pharmacol. 2000;130:289-98

Ebbert J, Jensen M. Fat depots, free fatty acids, and dyslipidemia. Nutrients. 2013;5(2):498-508.

Fasshauer M, Klein J, Neumann S, et al. Hormonal regu- lation of adiponectin gene expression in 3T3-L1 adipocytes. Biochem Biophys Res Commun. 2002;290(3):1084-9

Ference J and. Last A. "Choosing topical corticosteroids,” American Family Physician, 2009; 79 (2) 135-140,

Grzanka A, Misiołek M, Golusiński W, Jarząb J. Molecular mechanisms of glucocorticoids action: implications for treatment of rhinosinusitis and nasal polyposis. Eur Arch Otorhinolaryngology. 2011;268:247-53.

Hanoch C, Karen K, Kaushik C, et al. Glucocorticoids regulate transcription of the gene for phosphoenolpyru- vatecarboxy kinase in the liver via an extended glucocorticoid regulatory unit. J Biol Chem. 2005;280 (40):33873-84

Roujian L, Xiang Z, Juan L et al. Faculty Opinions recommendation of Genomic characterization and epidemiology of 2019 novel coronavirus: implications for virus origins and receptor binding. National Library of Medicine 2020; 395(10224):565-574.

Huang C, Wang Y, Li X, et al. Clinical features of patients infected with 2019 novel coronavirus in Wuhan, China. Lancet. 2020;395:497-506.

Lecoq L, Vincent P, Lavoie-Lamoureux A, Lavoie JP. Genomic and non-genomic effects of dexamethasone on equine peripheral blood neutrophils. Vet Immunol Immunopathol. 2009;128(1-3):126-31.

Li F, Li W, Farzan M, Harrison SC. Structure of SARS coronavirus spike receptor-binding domain complexed with receptor. Science. 2005; 309 (5742):1864-8.

Li Q, Guan X, Wu P, et al. Early transmission dynamics in Wuhan, China, of Novel coronavirus-infected pneumonia. N Engl J Med. 2020;382(13):1199-207 
Ling Y, Xu S., Lin Y et al., Persistence and clearance of viral RNA in 2019 novel coronavirus disease rehabilitation patients. Chin. Med. J. 2020133 (9) 1039-1043

Lippi G., Simundic A.M., Plebani M. Potential preanalytical and analytical vulnerabilities in the laboratory diagnosis of coronavirus disease 2019 (COVID-19) Clin. Chem. Lab. Med. 2020 25;58(7):1070-1076 doi: 10.1515/cclm-2020-0285.

Liu J, Weller GE, Zern B, et al. Computational model for nanocarrier binding to endothelium validated using in vivo, in vitro, and atomic force microscopy experiments. Proc Natl AcadSci U S A 2010;107: 16530-16535.

Mitre-Aguilar I, Cabrera-Quintero A, Zentella-Dehesa A. Genomic and non-genomic effects of glucocorticoids: implications for breast cancer. Int J ClinExpPathol. 2015;8(1):1-7

Muro S, Garnacho C, Champion J, et al. Control of endothelial targeting and intracellular delivery of therapeutic enzymes by modulating the size and shape of ICAM-1-targeted carriers. Molecular Therapy 2008;16: 1450-1458.

Nalayanda D, Fulton W, Colombani P. Pressure induced lung injury in a novel in vitro model of the alveolar interface: Protective effect of dexamethasone. J Pediatr Surg. 2014;49:61-5.

Newman S, Flower R, Croxtall J. Dexamethasone suppression of IL-1 $\beta$ induced cyclooxygenase 2 expression is not mediated by lipocortin-1 in A549 cells. Biochem Biophys Res Commun. 1994;202:931-9.

Oliver B, Robinson P, Peters M, Black J. Viral infections and asthma: an inflammatory interface? Eur Respir J. 2014;44(6):1666

Peckett A, Wright D, Riddell M. The effects of glucocorticoids on adipose tissue lipid metabolism. Metabolism. 2011;60 (11):1500-10

Ramamoorthy S, Cidlowski J. Corticosteroids-mechanisms of action in health and disease. Rheum Dis Clin N Am. 2016;42(1):15-31

Russell C, Millar J, Baillie J. Clinical evidence does not support corticosteroid treatment for 2019-nCoV lung injury. Lancet. 2020;395(10223):473-5. 
Saghazadeh A and Rezaei N. Towards treatment planning of COVID-19: rationale and hypothesis for the use of multiple immunosuppressive agents: Anti-antibodies, immunoglobulins, and corticosteroids. Int Immuno pharmacol. 2020;84:106560.

Saraya $\mathrm{M}$ and Amal A. Dexamethasone as adjunctive therapy for treatment of varicella pneumonia. Egyptian J Chest Dis Tuberculosis. 2012;61(3):9-13.

Sato T, Hayashi H, Hiratsuka M, Hirasawa N. Glucocorticoids decrease the production of glucagon-like peptide-1 at the transcriptional level in intestinal L-cells. Mol Cell Endocrinol. 2015;406:60-7

She J, Jiang J, Ye L, et al. Novel coronavirus of pneumonia in Wuhan, China: emerging attack and management strategies. Clin Trans Med. 2020;9:19-17. doi: 10.1186/s40169-02000271-z

Siddell S, Anderson R, Cavanagh D, et al. Coronaviridae. Intervirology 1983;20,181-189.

Singh A, Majumdar S, Singh R, Misra A. Role of corticosteroid in the management of COVID19: a systemic review and a clinician's perspective. Diabetes Metab Syndr. 2020;14(5):971978.

Singhal T. A Review of Coronavirus Disease-2019 (COVID-19). Indian J Pediatr. 2020;87(4):281-286.

Su S, Wong G, Shi W, et al. Epidemiology, genetic recombination, and pathogenesis of coronaviruses. Trends Microbiol 2016;24, 490- 502

Vabret V and Watmough J. Further notes on the basic reproduction number. In Mathematical Epidemiology, 2008; 159- 178.

Van W, Lutter R, Biezeveld M, et al. Effect of dexamethasone on tracheal viral load and interleukin-8 tracheal concentration in children with respiratory syncytial virus infection. Pediatr Infect Dis J. 2003; 22 (8): 721-6. 
Waljee K Mary A, Paul L, e.tal. Short term use of oral corticosteroids and related harms among adults in the United States: population based cohort study- National library Medicine 2017; 12(357) 1415

Wan Y, Shang J, Graham R, Baric RS, Li F. Receptor recognition by novel coronavirus from Wuhan: an analysis based on decade- long structural studies of SARS coronavirus. J Virol. 2020;94: e00127-0

Wang ZN, Zhang DW, Li X, et al. A novel coronavirus from patients with pneumonia in China, 2019. N Engl J Med. 2020;382(8):727-33.

Yang Z, Liu J, Zhou Y, et al. The effect of corticosteroid treatment on patients with coronavirus infection: a systematic review and meta-analysis. J Inf Secur. 2020; 81(1):13-20

Zhong J, Tang J, Ye C, Dong L. The immunology of COVID-19: is immune modulation an option for treatment? Lancet Rheumatol. 2020;2: 428-36.

Zoorob RJ, Cender D. A different look at corticosteroids. Am Fam Physician. 1998;58(2):44350.

Copyright (C) 2022 Widad M. Al-Bishri, Ghadeer H. Alhazmi, Barah M. Sulaiman, Duaa M. Mandeeli, Bashayer F. AlSulaymani and Adyan k. Qurban, AJRSP. This is an Open-Access Article Distributed under the Terms of the Creative Commons Attribution License (CC BY NC)

Doi: doi.org/10.52132/Ajrsp.e.2022.34.2 